\title{
Treadmill training with partial body weight support after stroke: A review
}

\author{
Stefan Hesse* \\ Klinik Berlin, Department of Neurological Rehabilitation, Charité - University Medicine Berlin, Germany
}

\begin{abstract}
Restoration and improvement of gait after stroke are major aspects of neurorehabilitation. Mobilization out of the bed into the wheelchair and verticalisation with the help of a standing frame are first steps. With the patient cardiovascular stable, gait restoration is put on the agenda. Instead of tone-inhibiting and gait preparatory maneuvers, patients should practice complex gait cycles repetitively. Treadmill training with partial body weight support enables the harness-secured patients to practice numerous steps assisted by two or three therapists. In controlled studies, it proved equally effective as walking on the floor. Gait machines, as the Lokomat or the Gait Trainer GTI, intend to relieve the strenuous effort for the therapists. For the GTI, several controlled trials showed a superior effect in acute stroke patients with respect to walking ability and velocity. For the ambulatory patient, aerobic treadmill training is effective to improve speed and endurance without worsening gait quality. Belt velocity and inclination are gradually increased so that the patients reach a predefined target heart rate. On the belt, patients walk more symmetrically, and higher velocities result in a facilitation of paretic muscles and render gait more efficient. In summary, gait rehabilitation has seen dramatic changes over the last years. More is to be expected.
\end{abstract}

Keywords: Gait training, treadmill, stroke, partial body weight support, hemiparesis

\section{Introduction}

Stroke is a leading cause of disability and handicap. The annual incidence is approximately 180 and the prevalence approximately 250 patients per 100.000 inhabitants in the EC [43]. Approximately $90 \%$ of these suffer from persisting motor deficits leading to disability and handicap, namely dependence in their daily activities, impaired arm and hand function and impaired walking ability.

Restoration of gait is a major goal for both patients and therapists in neurological rehabilitation following stroke, its outcome is decisive for the aspired social and vocation reintegration. Three months after stroke, $25 \%$ of the surviving patients are still wheelchair dependent, and in $60 \%$ the gait velocity and endurance are considerably reduced [39] so that patients have difficulties to

* Address for correspondence: Stefan Hesse, MD, Klinik Berlin, Kladower Damm 223, 14089 Berlin, Germany. Tel.: +49 3036503 105; Fax: +49 3036503 123; E-mail: bhesse@zedat.fu-berlin.de. cross a street in due time and have to stop after several hundred of meters due to exhaustion.

During the course of gait rehabilitation, three phases can roughly be distinguished: early mobilization out of the bed into the wheelchair, restoration of an independent gait, and then improvement of gait quality and function to meet the requirements of daily life.

\subsection{The early mobilization phase}

In the early phase after stroke onset, mobilization over the edge of the bed into the wheelchair and verticalisation with the help of a standing frame (Fig. 1) as soon as possible are generally accepted. The bed-ridden patient knows many medical complications (pneumonia, thrombosis etc), with the wheelchair the patient can leave his room again, either being pushed or actively, as a first step towards mobility. The vertical position in the standing frame is beneficial for almost everything from balance, cardiovascular reconditioning, prophylaxis of pneumonia, ulcera and contractures, stimulation of bowel and bladder to psychology. 


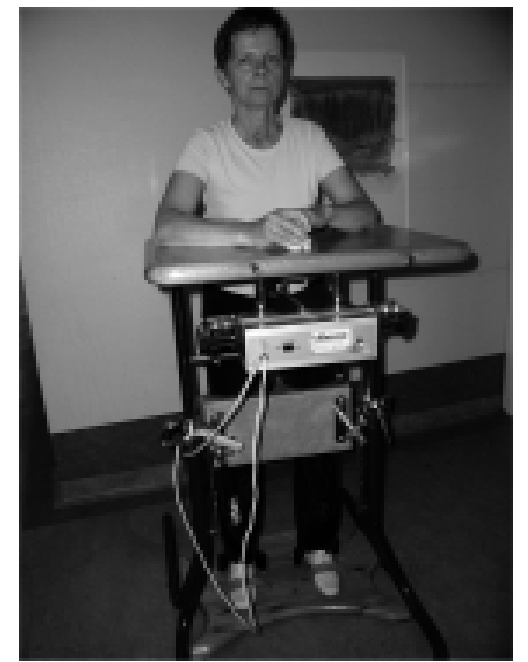

Fig. 1. Left hemiparetic stroke patient practising stance with the help of a standing frame.

The early mobilization is an integral part of the positively evaluated concept of stroke units [25] consisting of a multidisciplinary rehabilitation approach.

\section{The restoration of an independent gait}

With the patient in a stable cardiovascular situation, sustaining a prolonged verticalisation in the standing frame without relevant drop of the systolic blood pressure for approximately 5 to $10 \mathrm{~min}$, gait restoration is put on the agenda. When asked for their wishes at this stage, patients name independent walking as one of the most important for them.

Conventional gait therapy to reach this goal can be described as following one of four approaches: 1) Aggressive mobilization using brace, walking assist device and physical assistance by the therapist; 2) The Brunnstrom technique encouraging the use of synergistic movements; 3) Proprioceptive Neuromuscular Facilitation (PNF) technique encouraging the use of spiral and diagonal movements; 4) Neuro-Developmental Therapy (NDT, Bobath) using reflex inhibitory movements. Although different, none of these techniques have been proven to be superior to the others [29]. Proponents of the NDT approach, however, argue that such studies have not adequately assessed quality and symmetry of movement.

We have assessed gait velocity, endurance, and symmetry in 156 chronic hemiparetic patients before and after a 4-week comprehensive NDT rehabilitation program, the most frequently used therapy concept in Eu- rope [12]. Gait symmetry reflecting gait quality served as the primary outcome variable. The mean time interval after stroke was 12.8 weeks. Surprisingly, neither gait function nor gait symmetry improved substantially. We noted that tone inhibiting maneuvers and gait preparatory tasks during sitting and standing were the primary focus of the NDT program (Fig. 2). Gait itself was practiced very little, rarely more than 50 steps per session. At the same time therapists instructed patients to walk slowly and in a controlled manner resulting in an ongoing interruption of the gait rhythm.

\subsection{Modern concepts of motor learning}

Modern concepts of motor learning, however, favor a task-specific repetitive training, i.e. "The best way to improve walking is to walk." Kwakkel et al. showed that the intensity of gait rehabilitation and its outcome were positively correlated [23], and Horn and colleagues stressed the positive effect of an early and more aggressive gait therapy after stroke [10]. Further, Winstein and coworkers reported that balance training while standing improved balance but not gait symmetry in hemiparetic patients [44]. Dean et al. instructed hemiparetic patients to train balance while sitting [8]. At the end of the study, weight distribution between both lower limbs when sitting had improved, whereas symmetry of force distribution while standing remained unchanged. A recent large Norwegian outcome study compared the Bobath program and a task-specific motor relearning program (MRP), as advocated by Carr and Shepherd [2], in 61 acute stroke patients [24]. The MRP group stayed fewer days in hospital and their improvement in general motor functions was significantly better than in the Bobath group confirming the benefit of a task-specific repetitive approach.

\subsection{Partial Body Weight Supported Treadmill Training (PBWSTT)}

Body weight supported treadmill training (Fig. 3) was one of the first translations of the task-specific repetitive treatment concept in gait rehabilitation after stroke [13], following reports that it had shown promise in treating paraparetic subjects $[9,42]$.

PBWSTT enables otherwise non-ambulatory hemiparetic subjects to repetitively practice complex gait cycles. Patients wear a modified parachute harness to substitute for deficient equilibrium reflexes. The moving treadmill elicits and reinforces complex stepping movements. The harness supports a proportion of their 


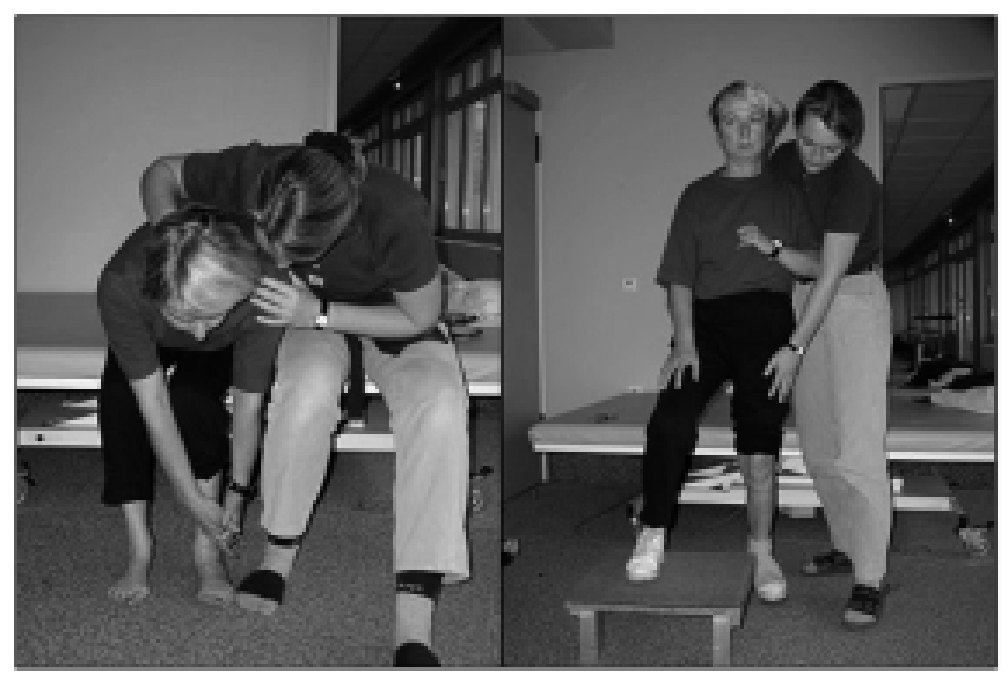

Fig. 2. Left hemiparetic stroke patient practising gait preparatory manoeuvres supported by a physiotherapist.

body weight so that subjects can carry their remaining body weight adequately, i.e. without knee collapse or excessive hip flexion during single stance phase on the affected leg.

The theoretical background of locomotor therapy is based on experiments in adult spinalized cats and incompletely lesioned primates. These studies show activation of presumed spinal and supraspinal gait pattern generators by locomotor therapy [26]. Adult spinalized cats, which do not regain locomotor ability spontaneously, relearn weight-bearing steps with their hind limbs following a several month training period on the treadmill.

Patients should be able to sit at the edge of the bed independently. Standing ability is not required. Cardiac risk factors, a history of recent deep vein thrombosis of the lower limbs, lower limb joint contractures and arthrosis can be limiting factors. Pusher Syndrome (i.e. pushing towards the affected side while standing and walking,) is not an exclusion criterion.

Initially two (or even three) therapists are required to assist the subjects' movements on the treadmill. Patients should practice stepping not only repetitively but in as smooth and normal a pattern as possible. One therapist, sitting alongside the patient, helps control the paretic foot and leg during the swing phase of gait and ensures that initial contact is made with the heel during the stance phase. The therapist also prevents hyperextension of the knee, and controls the symmetry of steps. A second therapist, standing behind the subject, assists weight shift onto the stance limb and promotes hip and trunk extension by applying firm pressure with the thumb on the rear of the pelvis or a flat hand on the chest. Patients are not allowed to sit in the harness as this prevents leg loading and results in continuous hip flexion. Based on animal experiments, alternating loading and unloading of the lower limbs, and hip extension during stance are the main peripheral drives needed to activate the gait pattern generator [10] Treadmill velocities of approximately $0.25 \mathrm{~m}$ per second and a body weight support of no more than $30 \%$ BW are initially recommended in non-ambulatory patients. During therapy, treadmill speed should be increased and body weight support reduced as soon as possible.

Biomechanical studies document that increasing BWS decreases the muscle activity of relevant weightbearing muscles in hemiparetic subjects [16]. Data suggest that BWS should not exceed $30 \%$ BW in order to optimize weight-bearing ability after stroke. Correspondingly, BWS should be reduced as soon as patients are able to carry their weight on the paretic limb without abnormal postures. BWS can be reduced when patients are able to sustain their body weight during single stance on the paretic limb, i.e. without knee buckling or "sitting" in the harness.

When PBWSTT with a mean of $15 \%$ BWS is compared with floor walking, hemiparetic patients randomized to PBWSTT walk more symmetrically, more dynamically and with better motor control. The dynamic electromyogram of shank muscles showed less premature activation of the gastrocnemius and more physiological activation of the tibialis anterior [15]. These results do not support the often-expressed fear of therapists that PBWSTT enforces repetition of an abnormal gait. On the contrary, patients walked more symmetrically with less spasticity during PBWSTT. Daniell- 


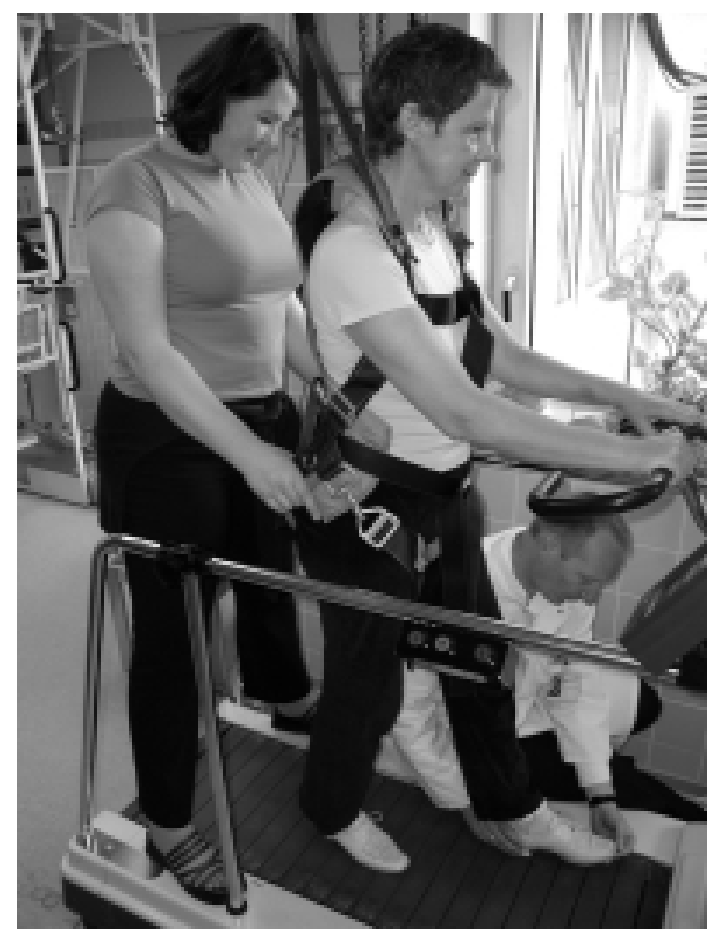

Fig. 3. Treadmill training with partial body weight support of a left hemiparetic stroke patient, assisted by two therapists.

son and Sunnerhagen compared oxygen consumption during treadmill walking with $30 \%$ BWS and without BWS in stroke patients. The $30 \%$ BWS condition required less oxygen consumption than full weight bearing. The authors concluded that patients with cardiovascular problems could tolerate treadmill therapy with BWS [6].

\subsection{Clinical evaluation of PBWSTT}

Initial studies [a baseline-treatment study $(n=9)$ and two single case-control studies following an A-BA design $(n=14)$ ] in chronic non-ambulatory hemiparetic subjects revealed that PBWSTT was superior to conventional physiotherapy with regard to restoration of gait and improvement of ground walking velocity [14] During the A-phases, treadmill therapy was applied alone or in combination with Functional Electrical stimulation (FES). Patients did not receive any additional conventional physiotherapy. During the Bphases, patients received conventional physiotherapy but no treadmill therapy. Each of the phases lasted 3 weeks. FES helped to facilitate movement on the treadmill. For instance, stimulation of the N. peroneus during the swing phase assisted with dorsiflexion of the foot. The results showed that patients improved their gait ability and over-ground walking velocity considerably during the first 3-week A-phase (A1) of daily treadmill training. During the subsequent period of three weeks of conventional physiotherapy (B) gait ability did not change, whereas the second A-phase further enhanced walking ability. All subjects who had been wheelchair-bound before therapy became ambulatory at least with verbal support by the end of the study. During one 30-minute session of treadmill training with PBWSTT, patients could practice up to 1000 gait cycles as compared with a median of less than 50 gait cycles during one regular physiotherapy session.

A large Canadian study of 100 acute stroke patients compared treadmill therapy with and without BWS [38]. Following randomization, 50 patients were trained to walk with up to $40 \%$ of their body weight supported (BWS-group), and the other 50 subjects were trained to walk with full weight-bearing on their lower limbs (no-BWS group). After a 6-week training period, the BWS group scored significantly higher than the no-BWS group for functional balance $(p=0.001)$, motor recovery $(p=0.001)$, over-ground walking speed $(p=0.029)$, and over-ground walking endurance $(p=0.018)$ (Fig. 3). A follow-up evaluation 3 months later revealed that the BWS group continued to have significantly higher scores for over-ground walking speed ( $p=0.006)$ and motor recovery $(p=0.039)$. 


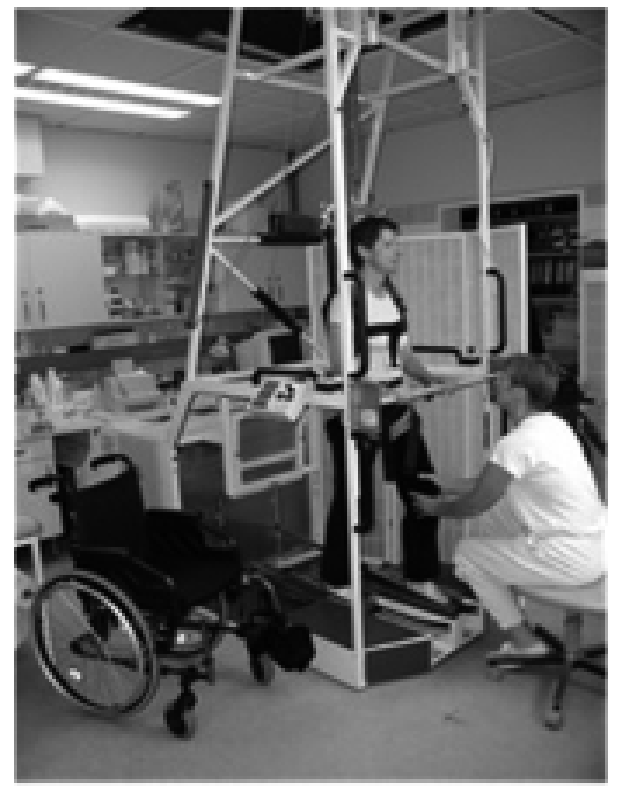

Fig. 4. Left hemiparetic stroke patient practising gait on the Gait Trainer GTI with partial body weight support assisted by one therapist.

Kosak and Reding conducted the first randomized study in 56 acute stroke patients who needed at least moderate assistance for walking [22]. The experimental group received treadmill therapy with PBWSTT following the above-mentioned principles. The control treatment consisted of aggressive early therapist-assisted ambulation using knee-ankle combination bracing and hemi-bar if needed, i.e. it did not follow conventional treatment concepts but stressed gait practice. Treatment session of up to $45 \mathrm{~min}$ per day, five days a week were given as tolerated for the duration of inpatient stay or until the patients could walk overground unassisted. The outcome of the two groups as a whole did not differ. However, a subgroup with major hemispheric stroke (defined by the presence of hemiparesis, hemianopic visual deficit, and hemihypesthesia) who received more than 12 treatment sessions showed significantly better over-ground endurance and speed scores favoring PBWSTT. The authors concluded that both approaches were equally effective except for a subset of severely disabled patients who were difficult to mobilize using physiotherapy alone.

Da Cunha and co-workers conducted a pilot study on 12 acute stroke patients either allocated to treadmill therapy or regular rehabilitation for 2 to 3 weeks [5] Dependent variables were cardiovascular performance on a bicycle exercise test and the locomotor subscore of the Functional Independence Measure (FIM-L). After intervention, the treadmill group performed better on the bicycle exercise test while the FIM-L did not differ.

Nilsson et al. presented a multicentre trial in hemiparetic patients at an early stage after stroke [32]. Three rehabilitation departments randomly allocated 73 patients to two groups who either received daily $30 \mathrm{~min}$ treadmill therapy or $30 \mathrm{~min}$ walking training overground according to the MRP program of Carr and Shepherd. During their time in the rehabilitation departments (about two months) all patients in the study received professional stroke rehabilitation in addition to walking training. There were no statistically significant differences between the groups at discharge or at 10month follow-up with regard to FIM, walking velocity, Fugl-Meyer Stroke assessment, or balance scores. The authors concluded that treadmill training with BWS at an early stage after stroke was a comparable choice to walking training over-ground. Correspondingly, a Cochrane report arrived at the same conclusion [31].

The treadmill community was stunned. Firstly, the control group had practiced gait repetitively on the floor assisted by the aggressive use of technical aids in accordance with the above-mentioned task-specific training concept. Secondly, the high effort for the therapists on the treadmill, e.g. to place the paretic limbs and to assist weight shifting, could have resulted in a too little therapy intensity to allow to discern between the two therapy modalities. Unfortunately, none of the trials reported exact numbers.

\subsection{Gait machines to relieve the strenuous effort of the therapists}

The major disadvantage of PBWSTT is the need for two or three therapists to assist with gait training of severely affected subjects limiting the number of steps practiced per session.

To relieve the strenuous effort for the therapists, gait machines as the Lokomat, the AutoAmbulator (www.healthsouth.com) and the Gait Trainer GT I, were designed. The Swiss Lokomat [3] consists of a treadmill and a powered exoskeleton with programmable drives flexing the hip and knee joints during the swing phase. The ankle joints are passively moved. With respect to controlled studies, one report, mentioned no difference between the therapy on the Lokomat and gait retraining on the floor in 30 acute stroke subjects randomized into two groups [21]. On the other side, a randomized crossover study, published in abstract form so far, adopted A-B-A respective BA-B design with only 16 subjects did find a superior 


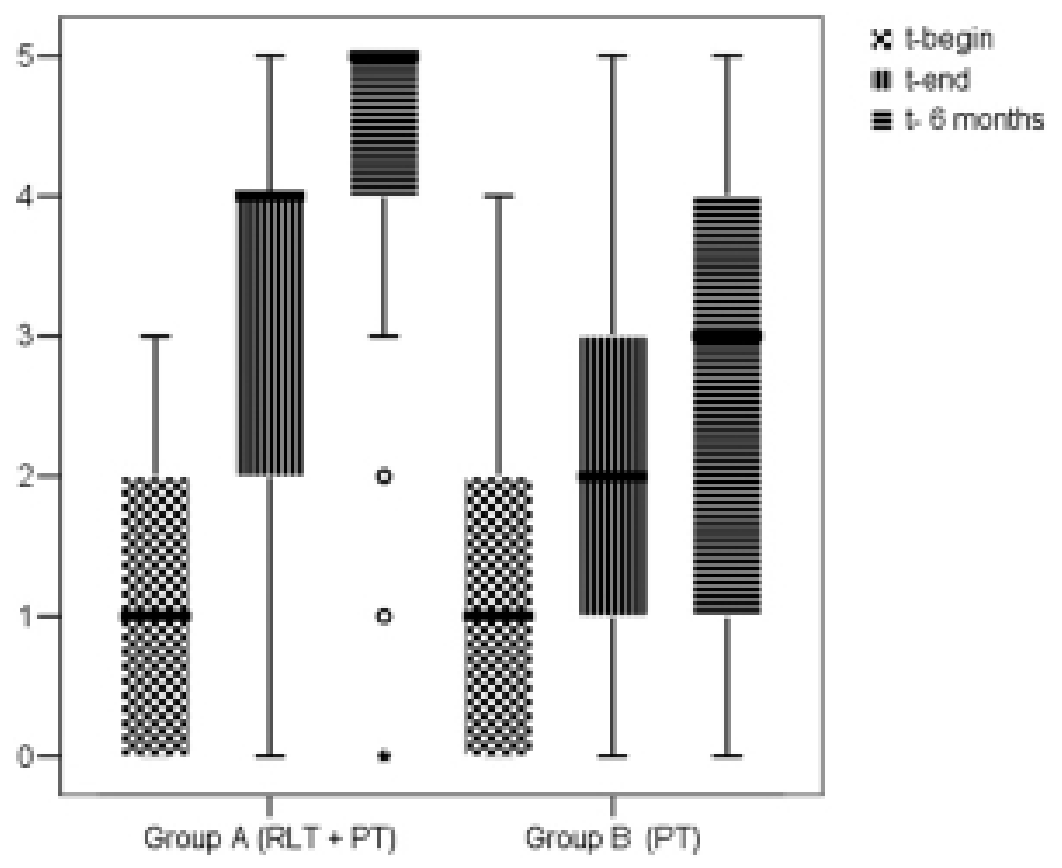

Fig. 5. Box plot of the Functional Ambulation Categories (FAC, 0-5) of patients of Group A ( RLT + PT, left, $n=77$ ) and Group B (PT, right, $n=78)$ at $\mathrm{T}_{\text {begin }}, \mathrm{T}_{\text {end }}$ and $\mathrm{T}_{6-\text { months. }}$ RLT $=$ repetitive locomotor training, $\mathrm{PT}=$ physiotherapy; 0 indicates values larger than one and a half of the box, * indicates values larger than three times of the box.

effect of the repetitive locomotor training [36]. With respect to muscle activation patterns of healthy subjects both on the treadmill and on the Lokomat, Hidler and colleagues described a reduced activity of the ankle flexor and extensor muscles throughout the gait cycle, and a higher, but not timely correct activation of the quadriceps muscle and hamstrings during the swing phase [19].

On the electromechanical GT I (Fig. 4), the harnesssecured patient is positioned on two foot plates, whose movement simulate stance and swing in a symmetric manner with a ratio of $60 \%$ to $40 \%$ between stance and swing [17]. The cadence and stride length can be adjusted within a speed range of 0.1 to $2.8 \mathrm{~km} / \mathrm{h}$ according to individual needs. A servo-controlled drive mechanism assists gait movements. Vertical and horizontal movements of the trunk are controlled in a phasedependent manner. Phase-dependent electrical stimulation of the quadriceps muscle during the stance phase helps to stabilize the knee; alternatively a therapist sitting in front of the patient can control knee movement.

Sagittal joint kinematics and dynamic electromyography of selected lower limb muscles in control subjects have been shown to closely mimic normal gait. Compared to over-ground walking, ankle dorsiflexion during the swing phase was less on the gait trainer (due to constructional constraints); correspondingly, patients hit the ground not with their heel but with the entire foot. Severely affected hemiparetic subjects need less help on the gait trainer than on conventional PBWSTT systems. Movement is more symmetric and the single stance phase of the paretic limb lasts longer on the gait trainer than on conventional PBWSTT systems. Dynamic electromyography of the lower limbs shows comparable activation of the trunk and thigh muscles. Plantar flexor spasticity is less on the gait trainer. Activation of the tibialis anterior muscle is, however, diminished on the gait trainer as the weight of the foot is partially carried by the footplate during the "swing phase." David et al. studied oxygen consumption of severely to moderately affected stroke patients while walking on the floor or on the gait trainer. On the GTI, the patients consumed less oxygen resulting in an increased walking time duration per session [7].

\subsection{Clinical evaluation of the Gait Trainer GT I}

Following an open study, an A-B-A and B-A-B study design compared locomotor therapy on the GT I (Aphases) and treadmill training with BWS (B-phases) in 30 non-ambulatory acute stroke subjects [40]. Each phase lasted two weeks. The therapeutic effort was 


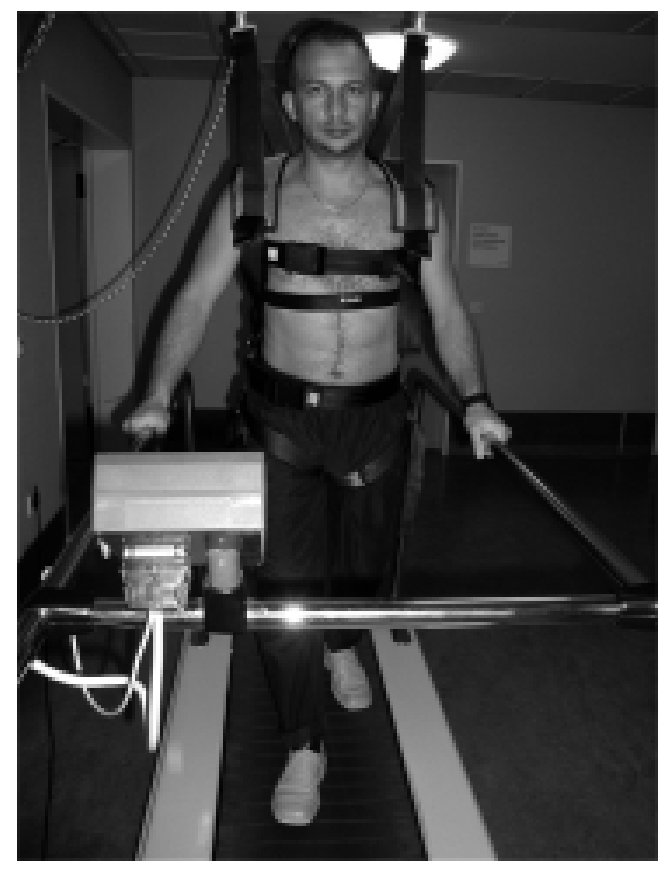

Fig. 6. Harness-secured aerobic treadmill training of a young hemiparetic patient. The heart rate is continuously monitored.

less on the gait trainer, with one instead of two therapists assisting the patients at study onset. During treatment, gait ability, gait velocity and lower limb motor functions improved in both groups. At the end of the treatment, significantly more patients could walk independently in the A-B-A group, 13 vs. 10. The median Functional Ambulation Categories (gait ability score, $0-5$ ) were 4 (3 to 4 ) in group A compared with 3 (2 to $3)$ in group B at the end of the treatment $(p=0.018)$.

Tong et al. included 50 non-ambulatory stroke subjects within six weeks after stroke onset. Patients were randomly assigned to 1 of 3 intervention groups: conventional gait training, gait trainer, and gait trainer in combination with FES on the machine. The intervention was a 20-minute session per day, 5 days a week for 4 weeks. In addition, all participants received their 40minute sessions of regular physical therapy every weekday. After the intervention, both locomotor groups had significantly more improvement than the conventional gait training group in walking speed, lower limb motor power, mobility and gait ability. No differences were found between the two locomotor groups [37].

A German multicentre trial (DEGAS, Deutsche Gangtrainerstudie) included 155 non-ambulatory stroke patients of four centers, the interval after a first supratentorial stroke before study onset ranged from 4 to 8 weeks [35]. Patients were allocated into two groups,
A and B. A-patients received $20 \mathrm{~min}$ GT I +25 min individual physiotherapy, B-patients $45 \mathrm{~min}$ individual physiotherapy every workday for 4 weeks. During their GT I session, patients practiced 800 to 1200 steps, the physiotherapy of both groups exclusively concentrated on gait therapy including the early use of technical aids. Both groups did not differ with respect to clinical data at study onset.

After treatment, significantly more patients of the locomotor group could walk independently: 44 out of 77 in group A vs. 19 out of 78 in group B. Responders were those patients who had reached a Functional Ambulation Category (0-5) of either 4 or 5, blindly assessed with the help of standardized videos (Fig. 5). Further, significantly more patients had reached a Barthel Index (0-100) of at least 75: 44 of 77 in group A vs. 21 of 78 in group B. At follow-up six months later, the positive effects in favor of the locomotor group persisted. For all secondary outcome variables, walking velocity, endurance, lower limb motor power and mobility, the locomotor group had improved significantly more during the intervention, in the period to follow-up the changes of all four variables did not differ between the two groups. Starting from a mean gait speed of $0.13 \mathrm{~m} / \mathrm{s}$ (group A) and $0.14 \mathrm{~m} / \mathrm{s}$ (group B) respectively at study onset, the patients of the locomotor (control) group reached a mean walking velocity of 0.44 $(0.32) \mathrm{m} / \mathrm{s}$ at the end of the intervention. Albeit statistically different, a mean absolute difference of $0.12 \mathrm{~m} / \mathrm{s}$ may be of limited clinical relevance. Side effects did not occur.

Positive results on non-ambulatory acute and chronic stroke patients have been reported by study groups in Korea, Slovenia and Portugal in abstract form. Several French centers currently conduct a multicentre trial, a Cochrane report on the effects of automated gait training in non-ambulatory patients is in preparation [30]. For 45 ambulatory chronic stroke patients, a Finish group compared training on the machine stressing a fast gait vs. a vigorous gait training program in and outside the clinic. Both methods were equally effective [33].

\section{The restoration of a fast and enduring gait meeting daily requirements}

With the patient having reached an independent gait at least on the ward or in his apartment, what are the next therapy goals? Therapists may name the improvement of gait quality in a first place. However, a large outcome study failed to show a substantial improve- 

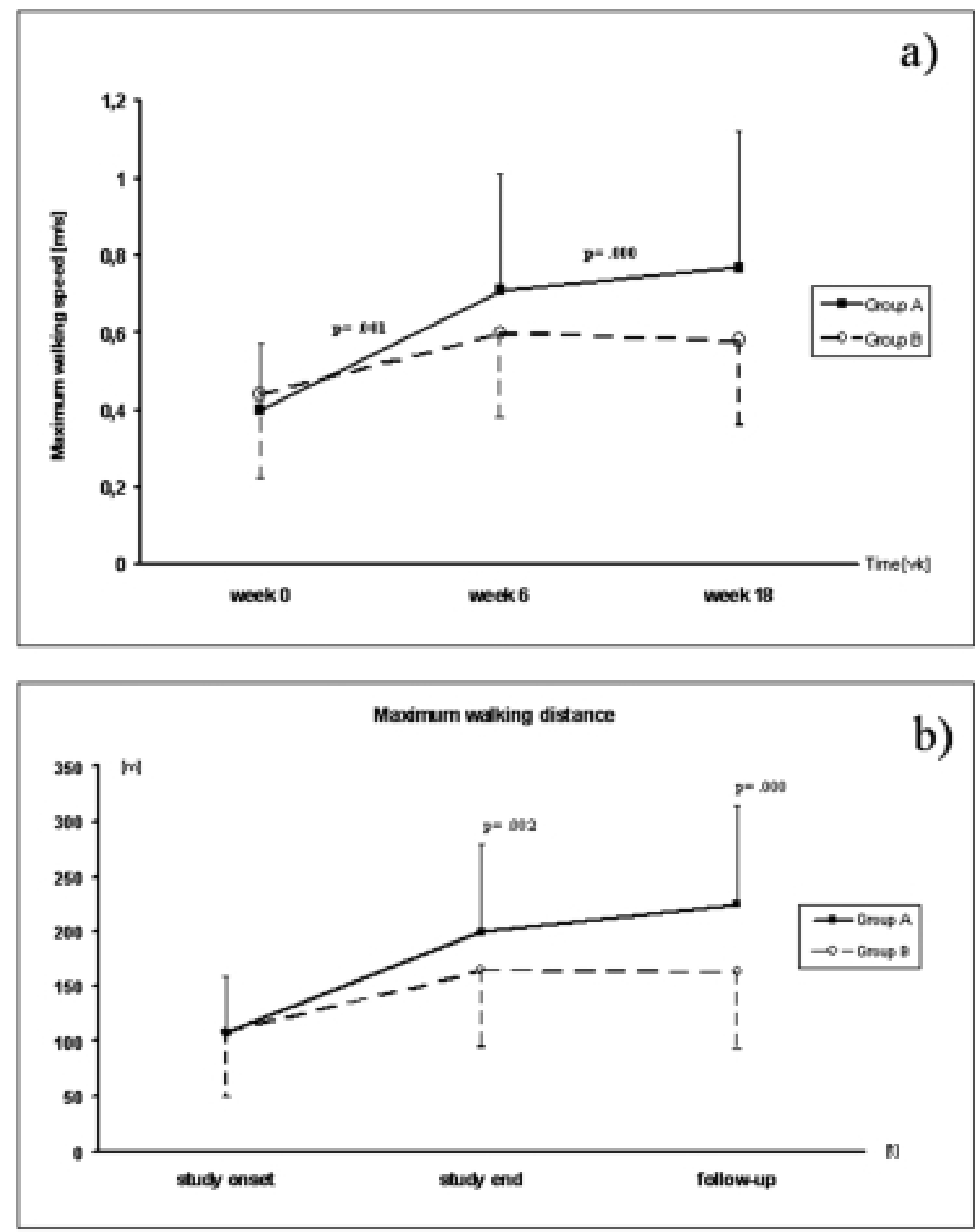

Fig. 7. Means and standard deviations for the aerobic treadmill group (closed squares) and the control group (open circles) at weeks 0,6 and 18 for a) maximum walking speed, and b) walking capacity.

ment of gait symmetry, serving as the processor variable of gait quality, after 4 weeks of Bobath therapy [12]. Further, professional groups and even individuals within one group can rarely agree on a common understanding of "gait quality" when assessing a single patient's walking pattern. Accordingly, the improvement of walking speed and endurance have emerged as equally important therapeutic goals in recent times of
ICF-oriented rehabilitation [4]. Although ambulatory, stroke patients cannot cross the street during the green phase of the traffic lights, have to stop after 200 to 300 meters, and do not climb more than 1 or 2 flights. Spasticity, poor motor control and fatigue surely contribute to this limited functional status, but rehabilitation professionals may have neglected the poor cardiovascular fitness and motor power of the stroke population. Fol- 
lowing the ictus, subjects have been immobilized for a long period, most of the patients suffer from concomitant cardiovascular diseases limiting fitness, and the hemiparesis prevents many kinds of active training. Further, Mackay-Lyons and Makrides showed that stroke patients rarely reached a heart rate sufficient to elicit a cardiovascular training effect during conventional physio or occupational therapy, nor could they substantially improve their exercise capacity after conventional rehabilitation [27].

Accordingly, aerobic endurance exercise training (not therapy, see definition of therapy as an improvement of coordination and training providing a repetitive suprathreshold stimulus to elicit adaptations of the cardiovascular and musculoskeletal system) is increasingly recognized as an important component in rehabilitation after stroke. Aerobic treadmill training with the patient harness-secured to prevent stumbling and falls, offers a perfect tool, already in use in cardiovascular rehabilitation (Fig. 6). Speed and inclination are adjusted so that the patients reach a predefined target heart rate to elicit a training effect. Higher gait velocities on the treadmill correlated with facilitation of relevant antigravity muscles without accompanying cocontraction of antagonist muscles in ambulatory stroke patients [18]. Further, patients walked more efficient at higher velocities, i.e. they consumed less energy per distance covered. These results support faster treadmill speeds [9] to facilitate activation of antigravity muscles, to economize the patients' gait and to train cardiovascular fitness. Some patients, however, may not be able to follow a high enough gait velocity to reach a target heart rate. The author's group therefore recommends to use a treadmill, not only equipped with a harness (prevention of falls), but also an inclination option. An additional inclination of up to $8 \%$ renders the gait more symmetric and less hurried, i.e. the patients take longer steps [41].

Eich et al. studied 50 patients with a stroke interval of less than 6 weeks, they were relatively young with a mean age of 62 years, cardiovascular stable and reached at least $50 \mathrm{~W}$ during bicycle ergometry. The experimental group practiced on the treadmill 30 min every workday for 6 weeks [11]. Treadmill speed and inclination were adjusted in a step-wise manner to achieve a heart rate of HR: (HRmax-HRrest) x 0.6 + HRrest. The control group received conventional physiotherapy. Improvements of walking velocity and capacity were significantly larger both at study end and at follow-up in the experimental group (Fig. 7). Side effects did not occur. Gait quality, assessed by an independent rater on maternity leave with the help of videos and a short gait quality chart asking for instance for the mode of initial contact etc., did not differ among groups.

For chronic patients with a stroke interval of more than 6 months, Macko et al. reported a similar result in favor of aerobic treadmill exercise. Their 61 patients had been randomized to 6 months ( $3 \mathrm{x} /$ week) progressive training or a reference rehabilitation program of stretching plus low-intensity walking [28]. Further, Pohl et al. had reported on the beneficial effect of a speed training on the treadmill in ambulatory stroke patients. They increased the treadmill speed in a step-like manner for short periods of time [34].

Group therapies consisting of a circuit training including treadmill training or treadmill training alone, have been positively evaluated in chronic ambulatory stroke patients $[1,8]$. Their advantage is the challenging group atmosphere, very similar to that of sport groups for cardiac patients.

\section{Summary}

In summary, gait rehabilitation after stroke has witnessed dramatic changes in the last 20 years: out of the bed into the wheelchair as soon as possible to be followed by a task-specific training concept with the repetition of numerous gait cycles on the treadmill with BWS or preferentially on gait machines.

With the patient independently walking, training of speed and cardiovascular fitness with the help of aerobic treadmill training have moved into the focus, both parameters are essential for participation in everyday life. Staying idle at home in the chair will definitely result in a deterioration of our patients' functional status.

Forthcoming studies will be needed to meet more and more the criteria of an evidence-based therapy program, and to clearly define in- and exclusion criteria. The future of gait rehabilitation may see more and more sophisticated machines to practise not only walking but also stair climbing and perturbations including the use of virtual reality. But the one-to-one physiotherapy session will remain the core enriched by intelligent machines and new concepts of efficient out-patient services, hopefully to the better of all participants.

\section{References}

[1] L. Ada, C.M. Dean, J.M. Hall, J. Bampton and S. Crompton, A treadmill and overground walking program improves 
walking in pesrons residing in the community after stroke: a placebo-controlled, randomized trial, Arch Phys Med Rehabil 84 (2003), 1486-1491.

[2] J. Carr and R. Shepherd, Neurological Rehabilitation, Oxford: Butterworth \& Heinemann, 1998

[3] G. Colombo, M. Wirz and V. Dietz, Driven gait orthosis for improvement of locomotor training in paraplegic patients, Spinal Cord 39 (2001), 252-255.

[4] A Courbon, P. Calmels, F. Roche and J. Ramas, FayolleMinon, Relationship between walking capacity and maximal exercise capacity, strength and motor defiency in adult hemiplegic stroke patients, Ann Readapt Med Phys 49 (2006), 614620.

[5] I.T. da Cunha Filho, P.A.C. Lim, H. Qureshy, H. Henson, T. Monga and E.J. Protas, A comparison of regular rehabilitation and regular rehabilitation with supported treadmill ambulation training for acute stroke patients, J Rehabil Res \& Develop 38 (2001), 37-47.

[6] A. Danielsson and K.S. Sunnerhagen, Oxygen consumption during treadmill walking with and without body weight support in patients with hemiparesis after stroke and in helathy subjects, Arch Phys Med Rehabil 81 (2000), 953-957.

[7] D. David, J.P. Regnaux, M. Lejaille, A. Louis, B. Bussel and F. Lofaso, Oxygen consumption during machine-assisted and unassisted walking : a pilot study in hemiplegic and healthy humans, Arch Phys Med Rehabil 87 (2006), 482-489.

[8] C. Dean and R. Shepherd, Task-related training improves the performance of seated reaching tasks following stroke: A randomised controlled trial, Stroke 28 (1997), 722-728.

[9] B.H. Dobkin, S. Harkema, P. Requejo and V.R. Edgerton, Modulation of locomotor-like EMG activity in subjects with complete and incomplete spinal cord injury, J Neurol Rehabil 9 (1995), 183-190.

[10] J. Duysens and K.G. Pearson, Inhibition of flexor burst generation by loading ankle extensor muscles in walking cats, Brain Res 187 (1980), 321-332.

[11] H.J. Eich, H. Mach, C. Werner and S. Hesse, Aerobic treadmill plus Bobath walking tarining improves walking in subacute stroke patients: a randomized controlled trial, Clin Rehabil $\mathbf{1 8}$ (2004), 640-651.

[12] S. Hesse, M.T. Jahnke, C. Bertelt, C. Schreiner, D. Lücke and K.H. Mauritz, Gait outcome in ambulatory hemiparetic patients after a 4-week comprehensive rehabilitation program and prognostic factors, Stroke 25 (1994), 999-1004.

[13] S. Hesse, C. Bertelt, A. Schaffrin, M. Malezic and K.H. Mauritz, Restoration of gait in non-ambulatory hemiparetic patients by treadmill training with partial body weight support, Arch Phys Med Rehabil 75 (1994), 1087-1093.

[14] S. Hesse, C. Bertelt, M.T. Jahnke, A. Schaffrin, P. Baake, M. Malezic and K.H. Mauritz, Treadmill training with partial body weight support as compared to physiotherapy in nonambulatory hemiparetic patients, Stroke 26 (1995), 976-981.

[15] S. Hesse, B. Helm, J. Krajnik, M. Gregoric and K.H. Mauritz, Treadmill training with partial body weight support: influence of body weight release on the gait of hemiparetic patients, $J$ Neurol Rehab 11 (1997), 15-20.

[16] S. Hesse, M. Konrad and D. Uhlenbrock, Treadmill walking with partial body weight support versus floor walking in hemiparetic subjects, Arch Phys Med Rehabil 80 (1999), 421-427.

[17] S. Hesse and D. Uhlenbrock, Development of an advanced mechanised gait trainer, controlling the movement of the centre of mass, for restoring gait in non-ambulant subjects, Biomed Tech 44 (1999), 194-201.
[18] S. Hesse, C. Werner, T. Paul, A. Bardeleben and J. Chaler, The influence of walking speed on lower limb muscle activity and energy consumption during treadmill walking of hemiparetic patients, Arch Phys Med Rehabil 82 (2001), 1547-1550.

[19] J.M. Hidler and A.E. Wall, Alterations in muscle activation patterns during robotic-assisted walking, Clinical Biomechanics 20 (2005), 184-193.

[20] S.D. Horn, G. DeJong, R.J. Smout, J. Gassaway, R. James and B. Conroy, Stroke rehabilitation patients, practice, and outcomes: is earlier and more aggressive therapy better? Arch Phys Med Rehabil 86 (2005), 101-114.

[21] B. Husemann F. Müller, C. Krewer, S. Heller and E. König, Effects of locomotion training with assistance of a robot-driven gait orthosis in hemiparetic patients after stroke: a randomized controlled pilot study, Stroke 38 (2007), 349-354.

[22] M.C. Kosak and M.J. Reding, Comparison of partial body weight-supported treadmill gait training versus aggressive bracing assisted walking post stroke, Neurorehab and Neural Repair 14 (2000), 13-19.

[23] G. Kwakkel, R.C. Wagenaar, J.W.R. Twisk, G.J. Lankhorst and J.C. Koetsier, Intensity of leg and arm training after primary middle- cerebral- artery stroke: a randomised trial, Lancet 354 (1996), 191-196.

[24] B. Langhammer and J.K. Stanghelle, Bobath or motor relearning programme? A comparison of two different approaches of pyhsiotherapy in stroke rehabilitation: a randomized controlled study, Clin Rehabil 14 (2000), 361-369.

[25] P. Langhorne, P. Dey, M. Woodman, L. Kalra, S. WoodDauphinee, N. Patel and E. Hamrin, Is stroke unit care portable? A systematic review of the clinical trials, Age Ageing 34 (2005), 324-330.

[26] R.G. Lovely, R.J. Gregor, R.R. Roy and V.R. Edgerton, Effects of training on the recovery of full-weight bearing stepping in the adult spinal cat, Exp Neurol 92 (1986), 421-435.

[27] M.J. Mackay-Lyons and L. Makrides, Cradiovascular stress during a contemporary stroke rehabilitation program: is the intensity adequate to induce a training effect? Arch Phys Med Rehabil 83 (2002), 1378-1383.

[28] R.F. Macko, F.M. Ivey, L.W. Forrester, D. Hanley, J.D. Sorkin, L.I. Katzel, K.H. Silver and A.P. Goldberg, Treadmill exercise rehabilitation improves ambualtory function and cardiovascular fitness in patients with chronic stroke: a randomized controlled trial, Stroke 36 (2005), 2206-2211.

[29] K.H. Mauritz, General Rehabilitation, Curr Opin Neurol 3 (1990), 714-718

[30] J. Mehrholz, C. Werner, J. Kugler and M. Pohl, Electromechanical-assisted training for walking after stroke, The Cochrane library Issue 4 (2006), CD 006185.

[31] A.M. Moseley, A. Stark, I.D. Cameron and A. Pollock, Treadmill training with body weight support for walking after stroke, Cochrane Database Syst Rev 3 (2003), CD002840.

[32] L. Nilsson, J Carlsson and A. Daniellson, Walking training of patients with hemiparesis at an early stage after stroke: a comparison of walking training on a treadmill with body weight support and walking training on the ground, Clin Rehabil $\mathbf{1 5}$ (2001), 515-527.

[33] S.H. Peurala, I.M. Tarkka, K. Pitkänen and J. Sivenius, The effectiveness of body weight-supported gait training and floor walking in patients with chronic stroke, Arch Phys Med Rehabil 86 (2005), 1557-1564.

[34] M. Pohl, J. Mehrholz, C. Ritschel and S. Rückriem, Speeddependent treadmill training in ambulatory stroek patients: a randomized controlled trial, Stroke 33 (2002), 553-558. 
[35] M. Pohl, C. Werner, M. Holzgraefe, G. Kroczek, J. Mehrholz, I. Wingendorf, G. Hölig, R. Koch and S. Hesse, Repetitive locomotor training and physiotherapy improve walking and basic activities of daily living after stroke: a single-blind, randomised multi-centre trial (Deutsche Gangtrainerstudie, DEGAS), Clinical Rehabilitation 21 (2007), 17-27.

[36] L. Saltuari, Efficiancy of Lokomat traing in stroke patients, $3^{\text {rd }}$ Joint Congress "Evidence-based medicine" in Neurorehabilitation, Zürich (2004), F11.

[37] R.K. Tong, M.F Ng and L.S. Li, Effectiveness of gait training using an electromechanical gait trainer with and without functional electrical stimulation, in subacute stroke: a randomized controlled trial, Arch Phys Med Rehabil 87 (2006), 1298-1304.

[38] M. Visintin, H. Barbeau and N. Korner-Bitensky, A new approach to retrain gait in stroke patients through body weight support and treadmill stimulation, Stroke 29 (1998), 1122 1128.

[39] D.T. Wade and R. Langton Hewer, Functional abilities after stroke: measurement, natural history and prognosis, J Neurol
Neurosurg Psychiatry 50 (1987), 177-182.

[40] C. Werner, S. von Frankenberg, T. Treig, A. Bardeleben and S. Hesse, Treadmill training with partial body weight support and an electromechanical gait trainer for restoration of gait in subacute stroke patients: a randomised cross-over study, Stroke 33 (2002), 111-118.

[41] C. Werner, A.R. Lindquist, A. Bardeleben and S. Hesse, The influence of treadmill inclination on the gait of ambulatory hemiparetic subjects, Neurorehab and Neural Repair $\mathbf{2 1}$ (2007), 76-80

[42] A. Wernig and D. Müller, Laufband locomotion with body weight support improved walking in persons with severe spinal cord injuries, Paraplegia 30 (1992), 229-238.

[43] G.R. Williams, J.G. Jingo, D.B. Matchar and G.P. Samsa, Incidence and occurrence of total (first-ever and recurrent) stroke, Stroke 30 (1999), 2523-2528.

[44] C.J. Winstein, E.R.Gradne, D.R. McNeal, P.S. Barto and D.E. Nicholson, Standing balance training: effects on balance and locomotion in hemiparetic adults, Arch Phys Med Rehabil 70 (1989), 755-762. 